\title{
ARTÍCULO
}

\section{Abrir la ciencia para cambiar el mundo}

\author{
Antonio Lafuente \\ Instituto de Historia, \\ Centro de Ciencias Humanas y Sociales, CSIC.
}

La noción de open science no sólo tiene que conjugar todas las formas de dar acceso (papers, data y notebooks), sino también las de promover participación, ya sea incentivando la colaboración entre una heterogeneidad de actores (science shops, citizen panels, consensus conferences, participatory action-research, living labs, hackerspaces, laboratorios ciudadanos, design assemblies) ya sea expandiendo el diálogo de saberes y haciendo más porosas las fronteras entre la academia y la urbe, los expertos y los amateurs, el conocimiento de laboratorio y el de campo, el aula y la plaza o el experimental y el experiencial. Abrir la ciencia también involucra el diseño de infraestructuras que garanticen la soberanía de la comunidad científica, como también abrir el ecosistema $o$, en otros términos, problematizar los protocolos que regulan la evaluación, financiación y licencia de la investigación, como también la gobernanza de la vida académica, incluidas las convocatorias y los jurados, o los premios, concursos, comisiones y, desde luego, los dispositivos de planeación.

Palabras clave:

Abrir contenidos, abrir contextos, abrir infraestructuras, abrir ontologías, abrir vida académica.

Open science is a new approach to the scientific process based on cooperative work, coupled to new tools for collaboration, and new routes for knowledge diffusion through online digital technologies. Open science entails a shift from the standard practice of publishing research results in scientific journals, towards sharing all available data and knowledge at the earliest stages of the research process. It requires a move from 'publishing as fast as possible' to 'sharing knowledge as early as possible'. Wilsdon et al (2017) 
Mucho se habla de lo abierto. Tanto, que abundan quienes sospechamos usos decepcionantes de una noción tan promisoria. Ahora entendemos mejor, como nos enseñó Elinor Ostrom, que lo abierto puede ser lo contrario de lo común, pues lo abierto, lo horizontal o lo transparente podrían ser los hilos que trenzan escenarios para la competición, el individualismo y la privatización (Haiven, 2016; Caffentzis \& Federici, 2014; Angelis, 2013; Wall, 2017). De ahí que, aunque sólo sea por motivos académicos, hay que desentrañar la noción de abierto y ver qué contiene. Necesitamos entender mejor su promesa (Albagli et al., 2015).

El concepto de open science tiene antecedentes en la obra de Merton y Popper, pero en su acepción actual, se parece más a lo que Paul David propuso en 2003 cuando argumentó la necesidad de hacer sinónimas las nociones de ciencia pública y ciencia abierta (David, 2003 y 2007; OCDE, 2015). Creo que hoy hablamos de ciencia abierta por dos motivos principales: uno, porque algo sucedió en la ciencia que la degrada $\mathrm{y}$, dos, porque el regreso a lo público, lo mundano y lo abierto quizás puedan ayudarnos a recuperar, como dice Michel Serres (1990), el último proyecto decente que le queda a Occidente.

Hay muchas maneras de entender lo abierto y algunas son contradictorias. Lo abierto mejora la calidad, la credibilidad y la universalidad de los procesos. Abrir la ciencia entonces es hacerla accesible, gratuita, mezclable y pública. También se hacen más eficientes, meritorias y competitivas las personas, físicas o jurídicas, que la practican. La ciencia, sus mundos y sus miembros, ganan con lo abierto (NASEM, 2018). Ganan popularidad, ganan prestigio, ganan dinero, ... y ganan poder. A las gentes de la ciencia les gusta ganar, son muy competitivas. La inmensa mayoría tiene hambruna de títulos, premios, citas. Su dependencia quizás sea insaciable. Tanto, que muchos observadores ven en la ciencia la vanguardia del neoliberalismo (Moulia et al., 2013). Y no les faltan motivos. Se insiste en que la apertura del conocimiento mejorará la carrera personal de quienes optaron por compartir (McKiernan et al., 2016). Un argumento que se repite hasta la saciedad. Ojalá no acabemos diciendo que compartir es la nueva forma de competir. $\mathrm{Y}$ es que, en efecto, el clamor por la ciencia abierta recorre un amplio espectro de posiciones, desde las que hablan de imperativo moral a las que la reclaman como parte de un modelo de negocio (Arza \& Fressoli, 2017; Fecher \& Ross-Hellauer, 2018; Roman, Liu \& Nyberg, 2018). No sorprende entonces que sean muchos los que piden un nuevo balance entre las derivas individuales y las colaborativas (Boettiger, 2017).

No tenemos más remedio que admitir que la precariedad del empleo en la academia, la competición extrema en los laboratorios, la privatización del conocimiento, las amenazas a la libertad de cátedra, el nuevo poder de los administradores y la deriva clientelar de los estudiantes, son síntomas inquietantes que transforman la comunidad universitaria en una comunidad de afectados. Una comunidad que está reaccionando ante la degradación de un bien público y de un bien común (Harney \& Moten, 2013). Degradación de lo público porque sólo tiene valor lo que IJESJP, 2020, V7, n2 
sabemos o queremos medir mediante prácticas de evaluación impuestas e importadas desde el mundo de los negocios. Degradación de lo común porque la competición, el individualismo y la meritocracia han condenado los cuidados, la pluralidad y el compromiso a la condición de prácticas resistencialistas y residuales (Kansa, 2014).

Abrir la ciencia entonces debiera tener que ver menos con abrir los contenidos que con abrir los contextos. El consenso parece ya imparable y todo apunta a que los papers, los datos y los cuadernos pronto serán mayoritariamente abiertos, especialmente tras la llamada crisis de la replicabilidad (Nosek, 2017). Pero hacer pública la ciencia también implicará medidas que favorezcan la participación ciudadana (Hecker, 2018), lo que es tanto como decir que la ciencia tiene que dejar de ser una especie de lenguaje privado y que los académicos, en consecuencia, tendrán que renunciar al rol de expertos, siempre más arrogante y distante de lo esperable, para admitir que la convivialidad es un bien imprescindible (Collier, 2017). Más aún, tendremos que reconocer que no es inevitable la falsa contradicción entre rigor y compromiso. Tenemos que aprender a vivir juntos, y eso implica poner menos énfasis en lo que nos une o nos separa y focalizarnos más en los que podemos hacer juntos. Tenemos que transitar desde el desiderátum de la objetividad de nuestros asertos a la objetualidad de nuestras acciones (Gherardi, 2008; Orlikowski, 2002; Knorr-Cetina, 1997). En fin, el mundo no necesita ser perfecto, ni verdadero, ni canónico, para ser compartido y habitable.

Abierto, ya lo dijimos, tiene que ver con lo accesible, lo participativo y, desde luego, con lo colaborativo y lo crítico. Es difícil imaginar una cultura abierta que no favorezca la diversidad de criterios y promueva el contraste libre de puntos de vista. Nada es fácil en el laboratorio. Los trabajadores de la prueba, como los llamó G. Bachelard (1998), tienen que esforzarse muy duro para conseguir datos fiables. Y por muy difícil que sea la labor académica, nada tiene que ver con el esfuerzo de pensar extramuros, a la intemperie, allí donde nunca funcionan los modelos, las abstracciones y las leyes. De hecho, el trabajo de campo siempre fue considerado una tarea de menor enjundia epistémica para un imaginario que soñaba con la ciencia como un placeless knowledge, una saber que parecía hacerse sin la mediación de cuerpos o sin arraigo local (Kohler, 2002). Abrir la ciencia también involucra incluir sus ineludibles vínculos con los fracasos, las incertidumbres, las expectativas y las emociones. Sería extraño, incluso engañoso, un uso de la palabra abierto para algo que no se relacione con lo tentativo, lo enraizado y lo inconformista. Esta perspectiva, nos invita a hablar menos de ciencia y más de investigación (Latour, 1998; Rheinberger, 2011; van Kerkhoff \& Lebel, 2006).

Lo abierto entonces tiene muchas capas y no pocos intentos de atraparla entre palabras (Vicente-Sáez \& Martínez-Fuentes, 2018). Tres son las capas con las que hasta ahora hemos trasteado: la de los contenidos, la de los contextos y la de las prácticas. Todas son importantes y todas tienen algo en común: remiten siempre a formas de vincular las cosas con los humanos. IJESJP, 2020, V7, n2 
Hasta aquí nuestra versión de lo abierto es, como diría B. Latour (1991), demasiado antropocéntrica (Star, 1999; Star \& Ruhleder, 1996; Edwards, 2013). Y por eso hay que dedicar unos minutos a discutir las infraestructuras de lo abierto. Hay que dedicar tiempo a pensar como impedir que el espacio público se convierta en una especie de terra nullius, abierta para el primero que llegue con capacidad de apropiársela (Hess \& Ostrom, 2003, Boyle, 2008). Y esta reflexión es urgente porque muchos piensan que cuando ya lo tengamos todo en abierto serán las grandes corporaciones de internet las que se apropiarán de la comunidad, igual que ya se están apropiando de nuestra privacidad y conciencia. Un mundo más abierto no necesariamente será más libre, ni más democrático, ni más transparente, ni más... hospitalario (Morozov, 2011). ¡Puede ser un infierno! Y algo tendremos que hacer para prevenir que la ciencia abierta no se convierta en la autopista que conduzca a una nueva especie de sumisión de toda la comunidad científica al dictum corporativo. Ya hemos perdido notables dosis de autonomía, y quizás sea esta amenaza lo que nos haga reaccionar. Una forma de hacerlo es hablar de infraestructuras (Schroeder, 2007). Y lo vamos a hacer de la mano de H. Lefebvre (1996), reclamando un novedoso derecho a infraestructuras (Corsín, 2013). Bien entendido que nunca caeremos en la tentación de considerarlas neutrales, pues abunda una literatura de naturaleza postcolonial que nos urge a construirlas inclusivas (Okune, 2018; Kera, 2017).

El espacio público no es un ente retórico e intangible. Es un ecosistema delicado que ensambla o asamblea multitud de dispositivos que lo sostienen (Estalella \& Corsin, 2016). Hasta la llegada del mundo digital teníamos un espacio público hecho de plazas, jardines, periódicos, aulas, tribunales, cafés, teatros y, desde luego, el sin fin de normas que regulan estos espacios. Nuestra idea de sociabilidad y buen vivir está asociada al correcto funcionamiento de esa trama institucional. La idea moderna de espacio público creció asociada a ciertas dicotomías que se nos han impuesto como necesarias, naturales y hoy discutibles como, por ejemplo, las de dentro/fuera, antes/después, pasado/futuro, individual/colectivo, actor/espectador, escritor/lector. La esfera pública digital nos ayudó a entender mejor estas restricciones y/o asimetrías. Y es normal que aquí nos preguntemos cómo la irrupción de la red puede afectar a la ciencia o, en otras palabras, como la avalancha de cachivaches digitales está transformando las condiciones de vida. Ambas esferas de lo público merecen atención. En la esfera ilustrada cabe preguntarse si podemos abrir las métricas que nos jerarquizan e incluir indicadores que nos ayuden a hacer visibles dimensiones de nuestro trabajo que están siendo desdeñadas. Y lo que vale para las métricas, puede extenderse a las formas de planificar, financiar y licenciar el conocimiento. Las sociedades científicas también podrían imaginarse menos corporativas e intentar conversaciones que incluyan el punto de vista de los legos y, desde luego, abrirse a encuentros con el país real que habitan, donde por cierto hay problemas cuya comprensión mejoraría implementando dispositivos de escucha y no solo de diagnóstico. Un porcentaje respetable de nuestro tiempo y atención debemos dedicarlo a los muchos organismos nacionales e internacionales dedicados a la IJESJP, 2020, V7, n2 
ciencia regulatoria y que, hasta ahora, casi milagrosamente, han logrado pasar desapercibidos, aun cuando ningún aspecto de nuestra vida personal esté fuera de su escrutinio. Nada que bebamos, respiremos, comamos, vistamos, escuchamos o imaginemos deja de ser regulado por un entramado de datos, criterios, directivas, comités, informes y discusiones que merecen y necesitan ser más transparentes, democráticas y contrastadas. Y, en fin, el espacio público se ensancharía si problematizáramos todos los mecanismos de gobernanza asociados a concursos, convocatorias, jurados, premios, seguimientos, plazas, nombramientos y, desde luego, gastos, agendas, comisiones, patrimonios y privilegios.

Sabemos que los protocolos con los que nos hemos dotado para gestionar nuestra vida en el espacio público pueden ser corrompidos o hacerse obsoletos, y por eso es tan recomendable como necesario abrirlos al escrutinio público. Y en esa tarea, la forma que demos a la capa digital de nuestras instituciones puede ser decisiva. Todos tenemos muchas esperanzas depositadas en blockchain, pues promete una red descentralizada, distribuida, inmutable, transparente y confiable (Bauwens \& Niaros, 2017; Wright \& De Filippi, 2015). Ahí es nada. No faltan tampoco los escépticos más descreídos (Golumbia, 2017; Luther, 2017; O’Dwyer, 2017; Levy, 2017). Blockchain representa la posibilidad de organizar la red de una forma totalmente autoregulada, compartida y fiable. Blockchain nos empuja a renovar nuestro entusiasmo por el utopismo digital $\mathrm{y}$, desde luego es mucho más que una moda hipster, ya sea que consideremos el elenco de instituciones que han apostado por su desarrollo, ya sea que valoremos el hecho de que en 2016 fracasaron 26.000 proyectos inscritos en GitHub basados en esta tecnología (Extance, 2017; Dovey, 2016).

También en ciencia comienzan a sonar un sinfín de proyectos esperanzadores (Brock, 2018). Y todos se adhieren a la noción de open science. No es este el lugar para describirlos. Necesitaríamos muchas páginas para esbozar semejante movilización. Soenke Bartling, fundador de Blockchain for Science (2016) ha lanzado un living document para quien quiera saber más o desee hacer aportaciones que nos ayuden a entender lo que está pasando y a construir el entorno que queremos habitar (Bartling, 2018). Las posibilidades son increíbles y no sólo en la ciencia académica, sino también en la llamada ciencia industrial (Friesike et al, 2015). Me detendré en algunas.

Blockchain permite monitorizar cualquier rastro que dejemos en la red y podríamos dar visibilidad y valor a las muchas actividades que son necesarias antes de que una contribución (texto, revisión, paquete de datos o recurso educativo abierto) llegue a la red. Los académicos podrían dejar atrás el temor a ser pirateados si trabajan en abierto $\mathrm{y}$, en consecuencia, hacer una apuesta firme por el trabajo colaborativo (Hurrell \& Meijer-Kline, 2011). Bastaría con asignar un valor (un token) al trabajo de compartir, evaluar, comentar, enlazar, citar o curar los contenidos de otrxs, ya sea interviniendo en plataformas o repositorios, ya sea contribuyendo a la difusión de IJESJP, 2020, V7, n2 
las ideas en redes sociales o en actividades presenciales (Ruseckiy, 2018; Sckor, 2018; Lewis, 2015). Los proyectos mismos pueden optar a una financiación descentralizada mediante la práctica del ICO (inicial coin offering, una especie de crowdfunding) que transforma a los promotores en accionistas y a quienes queríamos como pares en interesadas partes. Los administradores también podrían comenzar a evaluar más cosas que las citas, recursos o patentes obtenidas, contabilizando los likes o notifications que recibe la literatura gris, la participación en MOOC, el pronto impacto de sus recomendaciones o hipótesis, el page rank de los blogs que lo mencionan, el lugar donde influyen sus ideas, la diversidad de mundo donde tienen eco, la capacidad para conectar con la ciudadanía y las organizaciones de concernidos o las micro contribuciones ahora concebidas como procesos de brain storming. Y es que, en efecto, sigue habiendo muchas maneras informales e invisibilizadas de tener impacto (O'Dair \& Beaven, 2017; Wallach, 2014).

La propia ciudadanía, los amateur, podrían ser fácilmente incorporados y reconocidos, abriendo la posibilidad de un nuevo pacto social por la ciencia que tomara en cuenta la nueva fluidez entre el conocimiento que se produce y el que se demanda, o entre las técnicas de diagnóstico y el arte de la escucha o, en fin, la voluntad que tenemos de compartir y la necesidad de ser autores (Bartling \& Friesike, 2014). Abundan las pruebas de que la demanda de información y de datos existe, como lo prueba el Open Data for Developmen Network (OD4D), una web que muestra el uso de datos abiertos que hacen centenares de organizaciones ciudadanas en varias decenas de países. Son muchos los ejemplos que destacar. La selección que ha hecho Erin C. McKiernan (2017) es un buen comienzo y ayuda mucho a entender los motivos por los que las Universidades deben empezar reconocer y después premiar (con token, por supuesto) estas actividades.

Todavía es pronto para echar las campanas al vuelo, pero ya son muchas las instituciones que confían en esta tecnología (Peplow, 2016; Seebacher \& Schüritz, 2017). Ningún sector está siendo más activo que el de los biodata, pues blockchain convierte a los pacientes médicos en propietarios de datos que las Big Pharma quieren adquirir (Williams, 2018). Reconozco mi debilidad por los proyectos que promueve el CERN y, en particular, por las plataformas ORVIUM y ZENODO, basadas en el software libre, alojadas en servidores públicos y, en consecuencia, capaces de preservar la autonomía de la comunidad científica y la condición procomún del conocimiento (Hall, 2015). Y, para no dejarnos nada fundamental sin mencionar, tenemos que dejar una línea escrita a favor de la relación entre hardware libre e instrumentación científica, un problema acuciante cuando se mira desde el Global South y para el que viene reclamando atención Gathering for Open Science Hardware (Murillo, et al., 2017; Chagas, 2018; Ayass \& Serrano, 2012).

Abrir la ciencia debería contemplar también la posibilidad de profundizar sus encuentros con lo local, lo radical y lo experiencial. La deuda que tiene nuestro mundo con el activismo es IJESJP, 2020, V7, n2 
indiscutible. Los estudios de que disponemos han mostrado la presencia de los movimientos sociales en el mundo de la política, pero muy poco en el del conocimiento. No es difícil probar que los activistas también pueden y deben ser reconocidos como actores cognitivos (Hess, 2007; Lafuente, 2012). No es este el lugar para argumentarlo, aunque si lo sea para reivindicarlo. El activismo en la medida en la que transitó desde la protesta a la propuesta tuvo que diseñar la forma de responder nuevas preguntas con distintas prácticas metodológicas para el contraste, documentación, validación y difusión de sus respuestas. Lo más notable no es si fueron acertadas, sino la forma en la que fueron construidas, pues incorporaron puntos de vista vinculados al conocimiento local, campesino o indígena. Fueron capaces entonces de abrirse a la complejidad, no tanto de los algoritmos o los conceptos más sofisticados, sino de los encuentros menos codificables y más indisciplinares. Fueron capaces de utilizar el bien más abundante del planeta Tierra: la experiencia, ese saber arraigado a un territorio, a un cuerpo o a una idiosincrasia (Lafuente \& Estalella, 2015).

Tenemos centenares de casos documentados que describen movilizaciones ciudadanas críticas con el saber experto, tanto contra un diagnóstico como contra una terapia concreta $\mathrm{o}$, en términos más generales, contrarias a una política pública. Sabemos que estas manifestaciones de radicalismo por el medioambiente, por la paz, por la equidad, por la justicia, por la alimentación, por el trabajo, por la vivienda o por la salud, son un activo del que debemos aprender y poner en valor. Gran parte de lo que somos se lo debemos a quienes anónimamente donaron su tiempo, sus conocimientos y su energía por el bien común. Insisto, no los admiramos por su proyección política, sino que los reclamamos aquí por haber desbrozado el camino hacia los otros de la academia. Son verdaderos brokers sociales. Los necesitamos, como dice Isabel Stengers (2005), en la hora de las cosmopolíticas.

Incorporar lo local, lo radical y los experiencial es parte de la apertura que reclamamos en la ciencia. Y no es parte menor, ni tampoco diferible. No es fácil, pero alguna vez habrá que empezar. Para lograrlo, tenemos que apartarnos definitivamente de la rancia costumbre, entre los humanistas y gentes de letras, de creerse más críticos (o listos, o sensibles) y, a continuación, reprochar a los científicos su incapacidad para conectar con los asuntos mundanos y su inveterada costumbre de vivir distantes y distintos entre abstracciones incomprensibles. Nuestros distintos lenguajes o fraseos peculiares, como los llama Anna Tsing (2010), tienen que dejar de ser inconmensurables como ya se está haciendo en epigenética, neuroplasticidad o biodiversidad (Niewoehner, 2016), y admitir que las discusiones ontológicas deben dar paso a las simplemente ontográficas (Lynch, 2013); o, como diría Karen Barad (1999), asumir que la objetividad sólo es una responsabilidad colectiva asociada a los cuidados con los que revelamos el código que 
sustenta los procesos en los que participamos y no como una certeza epistémica nacida de intrincados malabarismos deductivos, discursivos e instrumentales (Heldke, 2001).

No lo hemos dicho todavía, aunque esté implícito en el texto: la open science tendrá que desplegarse en instituciones ancladas en formas más o menos jerárquicas, centralizadas y tecnocratizadas, que han (mal) acostumbrado a su personal a regímenes laborales tan competitivos e inestables, como impasibles ante la desigualdad, la injusticia y las asimetrías del entorno. La open science no pasará de ser un catálogo de buenos propósitos si no logra transitar hacia la open scholarship, lo que implica mucho más que la apertura de repositorios y bases de datos públicas, y reclama un cambio de cultura que atravesará todos los estamentos y estructuras académicas y administrativas de la Universidad (Jhangiani \& Biswas-Diener, 2017; Salmi, 2015; Ayris, 2018). Y hay dos escenarios probables: el primero será difícil pues las derivas meritocráticas de los profesores, tecnocráticas de los administradores y privatizadoras de los gobernantes auguran agrios desencuentros; el segundo, en cambio, imagina un escenario de convergencia que acabe con la antipática metáfora de la torre de marfil (Shapin, 2012) e instaure en la academia nuevos ensamblajes para lo abierto, lo colaborativo, lo experimental y lo mutualista. El conflicto no debería escindirnos en dos bandos antagónicos, sino que debiera servirnos para entender mejor los varios modelos coexistentes y la necesidad de una conversación que parta de un trabajo previo de documentación que no niegue la diversidad que somos.

Figura 1. Características de la ciencia abierta.

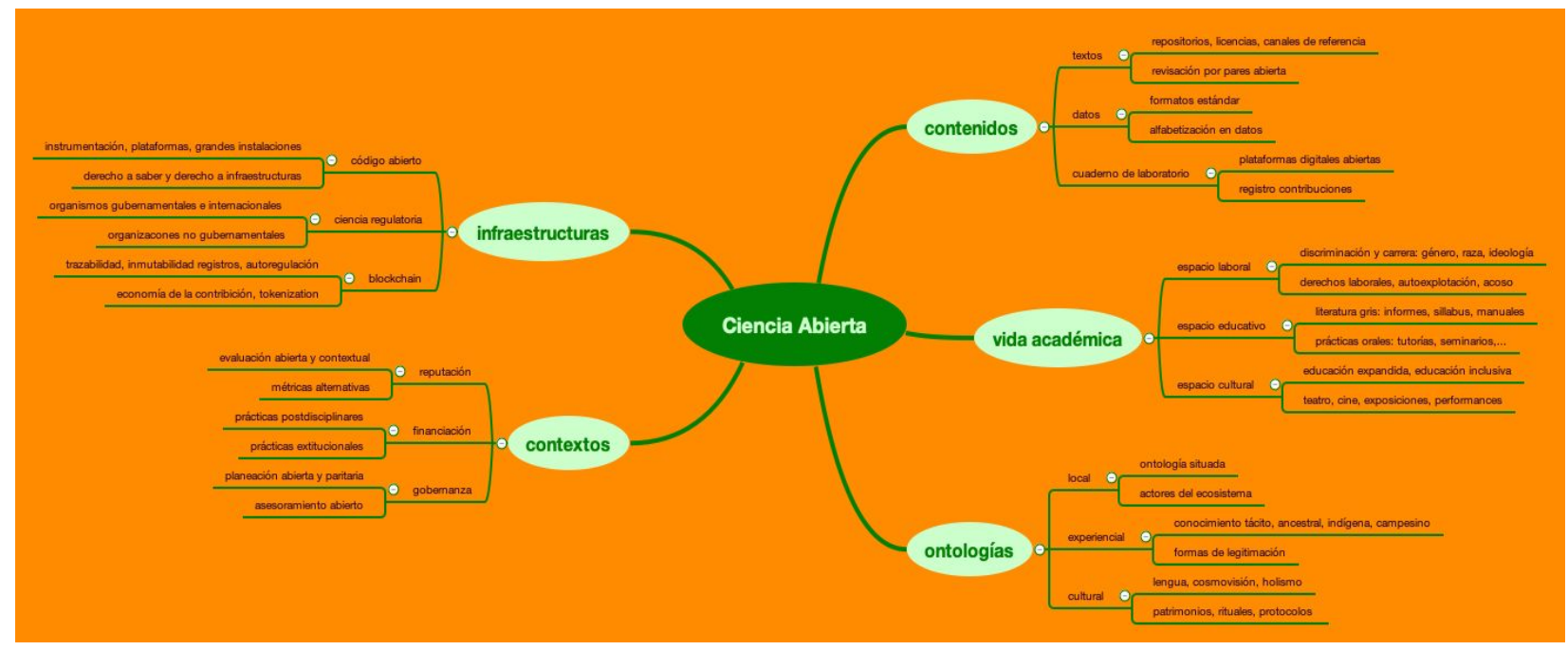

Fuente: Creación própia.

Antes que un campo de batalla necesitamos un ámbito de experimentación construido a partir de la situación real en la que nos encontramos. Hay plétora de declaraciones a favor de la ciencia IJESJP, 2020, V7, n2 
abierta. Muchos son los países y organizaciones internacionales que la promueven. Algunas comunidades nacionales o regionales van por delante de otras. La diferencia dentro de un mismo país o región pueden ser muy notables. Lo que nos separa, sin embargo, no es tan importante como la convicción de que la ciencia abierta es un proyecto de naturaleza internacional, interinstitucional y multicultural. La ciencia abierta es la nueva oportunidad que nos damos para hacer del conocimiento un bien común. Y lo cierto es que cada vez son más los científicos que se sienten incómodos, incluso maltratados, por los usos abusivos de la propiedad intelectual, el tufo abstracto de todo lo que importa en la academia y el daño infame que provocan las métricas. Quizás los profesores ya no se vean como una élite, sino parte de un colectivo magullado que renunció a sus ideales para preservar el empleo (Black, 2018). Cierta o no, la percepción dice mucho del cambio de paradigma cultural que necesitamos.

Nada puede ayudarnos más que disponer de un mapeo de los encuentros interdisciplinares (entre personas de lenguajes distintos) e indisciplinares (con personas de mundos incodificados), también nos ayudaría mucho conocer experiencias en las que se trabajó en grupo y luego se calificó individualmente. Seguro que ya hay muchas iniciativas de ciencia abierta y sería muy bueno documentarlas para saber cómo nacieron, qué dificultades enfrentaron o cuáles los criterios con los que fueron financiadas, reguladas y evaluadas. Ni que decir tiene que nos sería de gran utilidad conocer mejor las muchas formas de colaboración que se han dado entre la academia y la sociedad civil, lo que es tanto como preguntarnos por las formas particulares de organización, las prácticas innovadoras de investigación, las fuentes singulares de financiación y los modos alternativos de calificación. En resumen, un plan de ciencia abierta debiera comenzar por la creación de un nuevo ámbito de discusión que no parta de cero sino de toda la experiencia acumulada. $\mathrm{O}$, dicho con otras palabras, más que otra política científica o cultural, necesitamos otra cultura política.

\section{REFERENCIAS}

Albagli, Sarita., M.L. Maciel, \& A.H. Abdo, eds., (2015), Open Science, open issues, Brasília: Ibict, Rio de Janeiro: Unirio

Angelis, M de. (2013), Does capital need a commons fix? Ephemera, 13(3), 603-615.

Arza, Valeria \& Fressoli, Mariano (2017), "Systematizing benefits of open science", Information Services \& Use 37 (4): 463-474, 2017, DOI: 10.3233/ISU-170861, https://content.iospress.com/articles/information-services-and-use/isu 861 
Ayass, Myriam; Serrano, Javier (2012), "The CERN Open Hardware Licence", International Free and Open Source Software Law Review, 4 (1): 71-78. DOI: 10.5033/ifosslr.v4i1.65

Bachelard G (1998), Le rationalisme appliqué, Paris: PUF, 1998 (1re édition 1949).

Barad, Karen. 1999. “Agential Realism. Feminist Interventions in Understanding Scientific Practices”, in Mario Biagioli, ed., The Science Studies Reader, New York, NY: Routledge, pp. $1-11$.

Bartling, Sönke (2018), "Blockchain for Science and Knowledge Creation", Blockchain for science, https://www.blockchainforscience.com/2017/02/23/blockchain-for-open-science-the-living-docu $\underline{\text { ment/ }}$

Bartling, Sönke \& Friesike, Sascha, eds. (2014), Opening Science. The Evolving Guide on How the Internet is Changing Research, Collaboration and Scholarly Publishing, Cham: Springer, DOI 10.1007/978-3-319-00026-8

Bauwens, Michel \& Niaros, Vasilis (2017), "Value in the Commons Economy: Developments in Open and Contributory Value Accounting", Berlin: Heinrich Böll Stiftung; Amsterdam: P2P Foundation.

http://confrontations.org/wp-content/uploads/2016/12/1.Value-in-the-Commons-Economy_M.-B auwens.pdf

Black, A.L. (2018), "Reflecting on the Meaning of Academic Life", On Education. Journal for Research and Debate, 1 (3). https://doi.org/10.17899/on_ed.2018.3.4

Boettiger, Carl(2017) "Open Science: Balancing Individual Incentives with Common Good", Whitepaper written in response to seven questions on open science issued by invitation to the NSF/NIH ImagineU Conference (http://www.ncsa.illinois.edu/Conferences/ImagineU/, March 8-9, 2017). DOI: 10.5281/zenodo.268691,

Boyle, J (2008), The public domain: enclosing the commons of the mind, New Haven: Yale University Press.

Brock, Jon (2018), "Mapping the blockchain for science landscape", in Hackerdoon, consiltado: 1 octubre 2018 https://hackernoon.com/mapping-the-blockchain-for-science-landscape-546b61bfbd1 Caffentzis, George \& Federici, Silvia (2014), "Commons against and beyond capitalism", Community Development Journal, 49 (1): i92-i105, https://doi.org/10.1093/cdj/bsu006 
Chagas, A. M. (2018), "Haves and have nots must find a better way: The case for open scientific hardware", PLoS Biol 16(9): e3000014. https://doi.org/10.1371/journal.pbio.3000014

Collier S.J. (2017), "Neoliberalism and Rule by Experts", in Higgins V., Larner W. (eds) Assembling Neoliberalism, New York: Palgrave Macmillan, pp 23-43

Corsin, Alberto (2013), The right to infrastructure: a prototype for open source urbanism, http://digital.csic.es/bitstream/10261/85115/1/right infrastructure finalpreprint.pdf

David, P.A. (2003), "The economic logic of "open science" and the balance between private property rights and the public domain in scientific data and information: A primer", in P. Uhlir and J. Esanu, eds., National Research Council on the Role of the Public Domain in Science, National Academy Press, Washington, DC.

David, P.A. (2007), “The Historical Origins of Open Science”, Working paper, Stanford: Stanford University, Economics Dept. June, 1-82.

Dovey, Max (2016), "The Many Promises of Blockchain", MoneyLab 5, Consultado: 5 Octubre 2018, http://networkcultures.org/moneylab/2016/03/21/promise-of-the-blockchain/

Edwards, P. N. et al. (2013), Knowledge Infrastructures: Intellectual Frameworks and Research Challenges, Ann Arbor: Deep Blue. http://hdl.handle.net/2027.42/97552.

Estalella, A. and Corsin, A. (2016), "Matters of sense Preoccupation in Madrid's popular assemblies movement”, in Blok, A. \& Farias, I. eds., Urban Cosmopolitics: Agencements, assemblies, atmospheres. London, Routledge, pp. 147-163

Ethereum Foundation (2014), "DAOs, DACs, DAs and More: An Incomplete Terminology Guide", Ethereum Blog, 6 May 2014 [cited 8 oct 2018]. Available:

https://blog.ethereum.org/2014/05/06/daos-dacs-das-and-more-an-incomplete-terminology-guide/

Extance, Andy (2017), "Could Bitcoin technology help science?", Nature 552, 301-302, DOI: 10.1038/d41586-017-08589-4

Fecher, B \& Ross-Hellauer, T (2018), "Tautology, antithesis, rallying cry, or business model? "Open science" is open to interpretation", LSE Impact science blog, http://blogs.lse.ac.uk/impactofsocialsciences/2018/01/25/tautology-antithesis-rallying-cry-or-busi ness-model-open-science-is-open-to-interpretation/

Friesike, S., Widenmayer, B., Gassmann, O., \& Schildhauer, T. (2015), "Opening science: Towards an agenda of open science in academia and industry", Journal of Technology Transfer, 40(4), 581-601.

IJESJP, 2020, V7, n2 
Murillo, Luis Felipe R. Molloy,Jenny \& Dosemagen, Shannon (2017), Global Open Science Hardware Roadmap, http://openhardware.science/global-open-science-hardware-roadmap/

Gherardi, S. (2008) 'Situated Knowledge and Situated Action: What do Practice-Based Studies Promise?', in D. Barry and H. Hansen (eds) Sage Handbook of New Approaches in Management and Organization, pp. 516-25. London: Sage.

Golumbia, David (2017), The Politics of Bitcoin: Software as Right-Wing Extremism. By . Minneapolis, Minnesota: University of Minnesota Press

Grech, A. and Camilleri, A. F. (2017), "Blockchain in Education. Inamorato dos Santos, A. (ed.), Joint Research Centre (JRC), EUR 28778 EN, doi: 10.2760/60649

Hall, Gary (2015), "What does Academia_edu's success mean for Open Access? The data-driven world of search engines and social networking", LSE Impact blog, http://blogs.1se.ac.uk/impactofsocialsciences/2015/10/22/does-academia-edu-mean-open-access-i s-becoming-irrelevant/

Haiven M. (2016), "The Commons Against Neoliberalism, the Commons of Neoliberal-ism, the Commons Beyond Neoliberalism” (abstract), in S. Springer, K. Birch, J. Mac-leavy (eds.), Handbook of Neoliberalism (book proposal), New York, Routledge.

Harney, S., \& Moten, F. (2013). The undercommons: Fugitive planning \& blackstudy. Wivenhoe, New York and Port Watson: Minor Compositions.

Hecker, S. et al. (2018), Citizen Science: Innovation in Open Science, Society and Policy. London: UCL Press. https://doi.org/10.14324/111.97817873523

Heldke Lisa \& Kellert S. (1995), Objectivity as responsibility, Metaphilosophy, 26, 360-378.

Heldke, Lisa (2001), "How to be Really Responsible”, Nancy Tuana and Sandra Morgen (eds.), Engendering Rationalities, Albany: State University of New York Press, pp. 81-97

Hess, C and Ostrom, E. (2003), "Ideas, Artifacts, and Facilities: Information as a Common-Pool Resource" Law and Contemporary Problems 66(1/2): 111-145.

Hess, David (2007), Alternative Pathways in Science and Industry. Activism, Innovation, and the Environment in a Era of Globalization, Cambridge, The MIT Press

Hurrell C. and Meijer-Kline K. (2011), "Open access up for review: academic attitudes towards open access publishing in relation to tenure and promotion", Open Excess, 10 (2), 2011. 
Jhangiani, R S and Biswas-Diener, R (2017), Open: The Philosophy and Practices that are Revolutionizing Education and Science. London: Ubiquity Press. DOI: https://doi.org/10.5334/bbc

Kansa, Eric (2014), "It's the Neoliberalism, Stupid: Why instrumentalist arguments for Open Access, Open Data, and Open Science are not enough", LSE Impact blog, blog,http://blogs.lse.ac.uk/impactofsocialsciences/2014/01/27/its-the-neoliberalism-stupid-kansa/. Consultado: 8 oct 2018

Kera D (2017), "Science Artisans and Open Science Hardware". Bulletin of Science, Technology \& Society 37: 97-111

Kohler, Robert E. (2002), Landscapes and Labscapes: Exploring the Lab-Field Border in Biology, Chicago: Univ. Chicago Press.

Knorr-Cetina, K. (1997), Sociality with objects: Social relations in postsocial knowledge societies. Theory, Culture \& Society, 14(4): 1-30.

Lafuente, A. (2012), "Modernización epistémica y sociedad expandida." In R. Diaz (Ed). Educación expandida, Sevilla: Zemos98.

Lafuente, A. \& Estalella, A. (2015), "Ways of science: public, open, and commons", in S. Albagli, S., M.L. Maciel, \& A.H. Abdo (Eds), Open Science, open issues. Brasília: Ibict, Rio de Janeiro: Unirio

Lewis, A. (2015), "A gentle introduction to digital tokens", Bits on Blocks. Thoughts on blockchain technology, Consultado: 5 Octubre 2018, https://bitsonblocks.net/2015/09/28/gentle-introduction-digital-tokens/

Latour, B. (1991), "Technology is society made durable", in A sociology of monsters: essays on power, technology and domination Ed J Law (Routledge, London), pp. 103-131

Latour, B. (1998), "From the world of science to the world of research?", Science 280: 208-9

Lefebvre, H. (1996), "The right to the city", in Writings on Cities Eds E Kofman and E Lebas (Blackwell), pp 147-159.

Levy, Karen E. C (2017), "Book-Smart, Not Street-Smart: Blockchain-Based Smart Contracts and The Social Workings of Law", Engaging, Science, Technology, and Society 3: 1-15, DOI: 10.17351/ests2017.107

Luther, William J. (2017), David Golumbia's The Politics of Bitcoin: Software As Right-Wing Extremism (May 24, 2017). https://ssrn.com/abstract=2973059

IJESJP, 2020, V7, n2 
Lynch, Michael (2013), "Ontography: Investigating the Production of Things, Deflating Ontology", Social Studies of Science, 43(3): 444-462. doi:10.1177/0306312713475925.

McConaghy, Trent (2018), "Tokenize or die: Will industry giants be forced to dilute their power?" Brave New Coin, Consultado: 5 Octubre 2018, https://bravenewcoin.com/insights/tokenize-or-die-will-industry-giants-be-forced-to-dilute-theirpower

McKiernan, E.C. et al.(2016), "How open science helps researchers succeed", elife, 5 DOI: 10.7554/eLife. 16800

McKiernan E.C. (2017), "Imagining the 'open' university: sharing to improve research and education", PLoS Biol 15(10):e1002614. pmid:29065148

McCarthy, Jamesa \& Prudham (2004), Scott, Neoliberal nature and the nature of neoliberalism, Geoforum 35: 275-283, doi:10.1016/j.geoforum.2003.07.003

Milne, Gemma (2017), "Blockchain Science: An interview with Gemma Milne", Science Disrupt - Unblocked Events. In: Unblocked Events 1 Nov 2017 [cited 8 oct 2018]. Available: https://unblockedevents.com/2017/11/01/blockchain-science-interview-gemma-milne-science-dis rupt/

Moulia B. el al., (2013), "Main basse sur la science publique: Le «coût de génie» de l'édition scientifique privée", The Sigmoidal Journal 1: e0005

Morozov, Evgeny (2011), The Net Delusion. The Dark Side of Internet Freedom, NASEM, National Academies of Sciences, Engineering, and Medicine (2018), Open Science by Design: Realizing a Vision for 21st Century Research, Washington, DC: The National Academies Press. DOI: https://doi.org/10.17226/25116.

Niewoehner J. (2016) "Co-laborative anthropology: crafting reflexivities experimentally" in Jouhki J, Steel T, eds., Etnologinen tulkinta ja analyysi. Kohti avoimempaa tutkimusprosessia, Helsinki: Ethnos, pp. 81-124

Nosek, B A. (2017), "Opening Science” in Jhangiani, R S and Biswas-Diener, R. eds., Open: The Philosophy and Practices that are Revolutionizing Education and Science, London: Ubiquity Press, pp 89-99. DOI: https://doi.org/10.5334/bbc.g.

OCDE (2015), Making open science a realty, Paris: OCDE https://www.innovationpolicyplatform.org/system/files/OpenScience_FINAL_0.pdf 
O'Dair, M. \& Beaven, Z (2017), "The networked record industry: How blockchain technology could transform the record industry", Strategic Change 26: 471-80.

O'Dwyer, R. (2017), "Does Digital Culture Want to Be Free? How Blockchains Are Transforming the Economy of Cultural Goods", in R. Catlow, M. Garrett, N. Jones, and S. Skinner, eds., Artists Re:Thinking the Blockchain, Liverpool: Liverpool University Press.

Orlikowski, W. J. (2002) 'Knowing in Practice: Enacting a Collective Capability in Distributed Organizing', Organization Science 13(3): 249-73.

Okune, Angela el al. (2018), "Whose Infrastructure? Towards Inclusive and Collaborative Knowledge Infrastructures in Open Science", in Leslie Chan \& Pierre Mounier, eds., ELPUB 2018, Toronto, Canada. DOI: 10.4000/proceedings.elpub.2018.31, https://hal.archives-ouvertes.fr/hal-01816808

Peplow, Mark (2016), Distributed Ledger Technology: beyond block chain, London: Government Office for Science, https://assets.publishing.service.gov.uk/government/uploads/system/uploads/attachment_data/file 1492972/gs-16-1-distributed-ledger-technology.pdf

Rheinberger, H-J (2011), "Consistency from the perspective of an experimental systems approach to the sciences and their epistemic objects", Manuscrito 34(1): 307-321.

Roman, M.; Liu, J.; Nyberg, T. (2018), "Advancing the open science movement through sustainable business model development”, Ind. High. Edu. 32 (4), 226-234.

Ruseckiy, A. (2018), "How tokenization drives the new economy for science financial and reputational incentives, governance and other functions of tokens on DEIP", in DEIP, Consultado 4 Octubre 2018, https://medium.com/deip/how-tokenization-drives-the-new-economy-for-science-2ac21f62dflb

Sckor, A. (2018), "How to enable self-governance in decentralized science Delegated Proof of Expertise Contribution", DEIOP, Consulotado 4 Octubre 2018, https://medium.com/deip/delegated-proof-of-expertise-contribution-c6352241ff57

Seebacher S. \& Schüritz R. (2017), "Blockchain Technology as an Enabler of Service Systems: A Structured Literature Review", in Za S., Drăgoicea M., Cavallari M., eds.. Exploring Services Science. IESS 2017. Lecture Notes in Business Information Processing, vol 279. Springer, Cham

Schroeder, R. (2007), "E-research infrastructures and Open Science: Towards a new system of knowledge production?", Prometheus 25(1): 1-17.

IJESJP, 2020, V7, n2 
Stengers, Isabelle (2005), "The cosmopolitical proposal", in Bruno Latour y Peter Weibel, eds., Making things public: atmospheres of democracy, Cambridge, Massachusetts, and London: The MIT Press, pp. 994-1003.

Serres, Michel \& Latour, Bruno (1990), Conversations on Science, Culture and Time, Translated by R. Lapidus, University of Michigan Press,

Star, S L (1999), “The Ethnography of Infrastructure" American Behavioral Scientist 43(3) 377-391. DOI: 10.1177/00027649921955326

Star S. L. \& Ruhleder K. (1996), "Steps toward an ecology of infrastructure: Borderlands of design and access for large information spaces", Information Systems Research 7(1): 111-134.

Tsing, Anna (2010), "Worlding the Matsutake Diaspora." in Experiments in Holism, in Ton Otto and Nils Bubandt, eds., Chichester: Wiley-Blackwell, pp. 47-66.

van Kerkhoff L. \& Lebel L .(2006), "Linking knowledge and action for sustainable development”, Annu Rev Environ Resour 31: 445-477

Vicente-Saez R. \& Martinez-Fuentes C. (2018), “Open Science now: A systematic literature review for an integrated definition”, Journal of Business Research, 88, 428-436.

Wall, D. (2017), Elinor Ostrom's Rules for Radicals. London: Pluto Press

Wallach, D.S. (2014), "Bitcoin for Rockstars. How Cryptocurrency Can Revolutionize The Music Industry", Wired, 12.10.2014, Consultado: 1.102.018

Williams, Shawna(2018), "Startups Plan the Health Data Gold Rush", The Scientist (1 Oct 2018), https://www.the-scientist.com/bio-business/startups-plan-the-health-data-gold-rush-64840

Wilsdon, J., Bar-Ilan, J., Frodeman, R., Lex, E., Peters, I., \& Wouters, P. (2017). Next-generation metrics. Responsible metrics and evaluation for open science. Directorate-General for Research and Innovation (European Commission). Retrieved from http://doi.org/10.2777/337729

Wright, Aaron and De Filippi, Primavera (2015), "Decentralized Blockchain Technology and the Rise of Lex Cryptographia", SSRN, DOI:10.2139/ssrn.2580664, Consultado: 5 de octubre 2018, https://ssrn.com/abstract $=2580664$

IJESJP, 2020, V7, n2 